\title{
Traction patterns of tumor cells
}

\author{
D. Ambrosi - A. Duperray · V. Peschetola . \\ C. Verdier
}

Received: date / Revised: date

\begin{abstract}
The traction exerted by a cell on a planar deformable substrate can be indirectly obtained on the basis of the displacement field of the underlying layer. The usual methodology used to address this inverse problem is based on the exploitation of the Green tensor of the linear elasticity problem in a half space (Boussinesq problem), coupled with a minimization algorithm under force penalization. A possible alternative strategy is to exploit an adjoint equation, obtained on the basis of a suitable minimization requirement. The resulting system of coupled elliptic partial differential equations is applied here to determine the force field per unit surface generated by T24 tumor cells on a polyacrylamide substrate. The shear stress obtained by numerical integration provides quantitative insight of the traction field and is a promising tool to investigate the spatial pattern of force per unit surface generated in cell motion, particularly in the case of such cancer cells.
\end{abstract}

\section{Introduction}

Cell locomotion occurs through complex interactions that involve, among others, actin polymerization, matrix degradation, chemical signaling, adhesion and pulling on substrate and fibers [27]. When focusing on mechanical aspects only, a major issue is the determination of the dynamic action of the cells on the environment during migration: the cells adhere, pull on the surrounding matrix and move forward. As a cell can have more than one hundred focal adhesion sites, it is quite difficult to obtain a pointwise

D. Ambrosi

Dipartimento di Matematica, Politecnico di Torino, corso Duca degli Abruzzi 24, 10129 Torino, Italy

A. Duperray

(a) INSERM, U823, Grenoble, France. (b)Université Joseph Fourier-Grenoble I, Faculté de Médecine, Institut d'oncologie/développement Albert Bonniot et Institut Francais du Sang, UMR-S823, Grenoble, France

V. Peschetola and C. Verdier

Laboratoire de Spectrométrie Physique, CNRS and Université Grenoble I, UMR 5588, 140 avenue de la physique, BP 87, 38402 Saint-Martin d'Hères cedex, France 
description of the force per unit surface exerted by moving cells on a direct basis. Considerations of this kind suggest that the dynamics of cell locomotion can be fruitfully studied as an inverse problem, an idea that dates back to the seminal paper of Harris and coworkers [17]. A thin elastic film is deformed by cell traction into a wrinkled pattern and the size of the crimps is correlated to the shear load. Unfortunately, buckling of thin film is an essentially nonlinear phenomenon and a quantitative reconstruction of the exerted traction field would call for a non-trivial stability analysis in nonlinear elasticity.

A quantitative methodology that obviates such a problem has been proposed in 1996 by Dembo et al. [10], using pre-stressed silicone rubber, an approach further improved by Dembo and Wang in 1999 [9]. They deduce the traction exerted by a fibroblast on a polyacrylamide substrate from the measured displacement of several fluorescent beads merged in the upper layer of the gel. The gel is soft enough to remain in a linear elasticity regime and no wrinkles form.

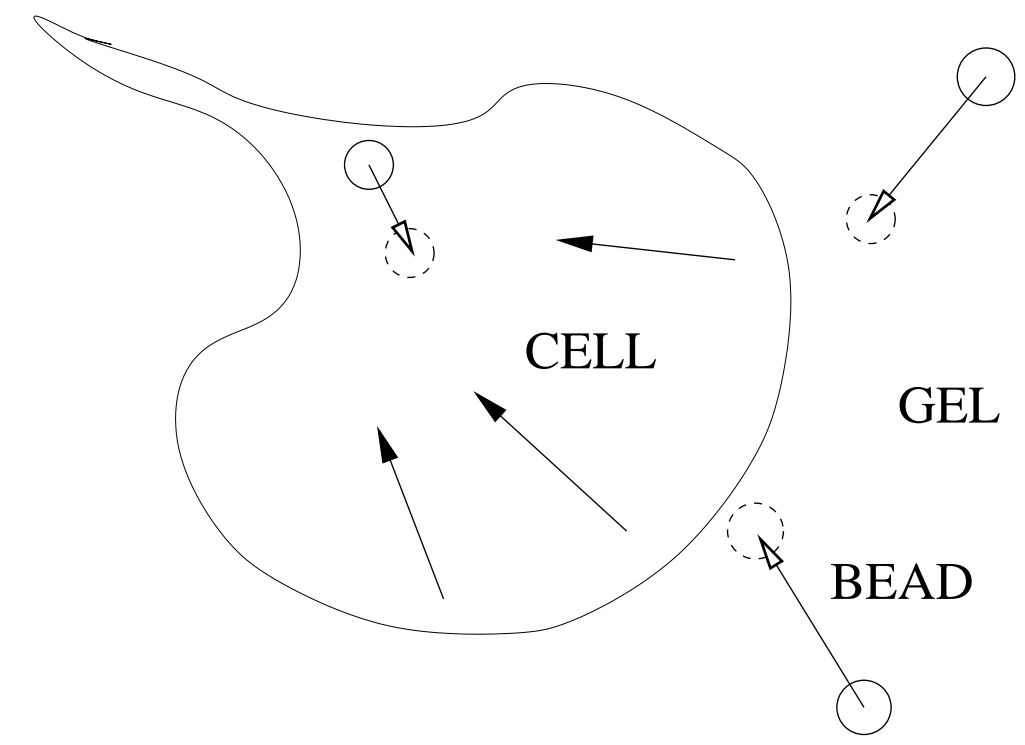

Fig. 1 The experiment by Dembo and Wang. The cell exerts a traction (filled-hat arrows) on the gel. The beads, embedded in the substrate, move from the former position (continuous-line circle) to the new one (dashed circles). The difference in these positions gives the displacement of the gel (empty-head arrows).

In a recent paper [2] the same biomechanical issue studied by Dembo and Wang has been addressed using a different mathematical approach, based on the classical functional analysis framework due to Lions [21]. The minimization of the distance between the measured and the computed displacement under penalization of the force magnitude is stated before the elasticity equations are solved [28]. Standard derivation of the cost function leads to two sets of elastic-type problems: the direct and the adjoint one. The unknown of the adjoint equation is just the shear stress exerted by the cells we are looking for. The two systems of equations are then solved numerically by a coupled finite element discretization.

In the present work, the adjoint method is applied to determine the traction field ex- 
erted by tumoral T24 cells on a polyacrylamide substrate of known mechanical properties. Our aim is to obtain a spatial detail of the tension field on substrates of different stiffness, and to compare the behaviour of the cells in such a varying environment (forces, displacements, migration velocities).

The paper is organized as follows. Methods for measuring traction forces are first presented Section 1.1, with emphasis on the classical methodology by Dembo and Wang detailed in Section 1.2. Then the adjoint method is summarized in Section 2. Materials and methods are detailed in Section 3. Section 4 contains the results of the computed shear stress field as exerted by a T24 cell on a flat surface. The last section is devoted to a comparison between the different methods including a discussion about the present results.

\section{Determination of traction forces}

\subsection{Methods for measuring traction forces}

Several methods are available to determine traction forces exerted by cells as they move on rigid substrates. They can be classified as follows:

- Wrinkles on elastomeric surfaces.

This is the original method proposed earlier by Harris [17] who showed that cells in contact with an elastic medium deform the latter, enabling the formation of wrinkles. The wrinkling patterns come from the large deformations of the substrate undergoing buckling. Previous observations first reported on the possibility to follow cell division using silicone-rubber substrates [5]. The method was further improved when coupled with DIC-microscopy to determine accurate forces as in the case of keratocyte migration [6]. This method is interesting but requires complex integration due to the nonlinearity of the buckling equations.

- Beads in an elastic matrix.

This is certainly the most popular method as originally proposed by Lee et al. [20] who used silicone substrates to study cell migration after inserting $1 \mu \mathrm{m}$-beads at the substrate surface. Calibration was achieved by looking at the bead motion while applying known forces with needles (whose deflection was measured). Using this idea, the authors determined the traction forces exerted by keratocytes, which are larger on the sides (around 20nN), due the special motion of such crawling cells. Using this concept, Dembo and Wang [9] used smaller fluorescent beads $(200 \mathrm{~nm}$ in size) and determined their positions as compared to the initial one, to obtain displacements. Then they solved the elasticity problem using the method which is presented in more details in the next part. Usually, polyacrylamide gels are used because their mechanical properties can be tuned (generally between $5-30 k P a$ ). Several issues have been addressed by these authors, in particular the 'durotaxis' problem [22], i.e. cells move from less rigid surfaces to more rigid ones, this being correlated with larger traction forces (i.e. stronger focal adhesions) on the rigid substrate. Another approach [26] has focused on the levels of forces exerted by endothelial cells over time during spreading, showing levels reaching around $8 \mathrm{kPa}$ after a few hours. This method can give a continuous description of the force field when carried out with the proper integration method.

- Regular arrays of microneedles - the 'fakir carpet'. 
A direct way to determine local forces was proposed by Galbraith and Sheetz [16] who developed a microsystem allowing small pillars/needles deflections to give access to local forces exerted by cells at the adhesion sites. The method was further improved by Balaban et al. [3] who measured stresses at focal adhesion sites (around $5 n N / \mu m^{2}$ ) on different micropatterned surfaces. Finally Tan et al. [33] engineered arrays of microneedles regularly spaced, which improved the accuracy of the method without further calculations. Using this tool, they showed that cell morphology controls the levels of forces exerted. Furthermore, it seems that cells adapt their forces [29] according to the substrate's rigidity in a linear manner. Finally the use of similar microneedles arranged in an anisotropic fashion proved that epithelial growth can be controlled by anisotropic rigidity [30]. Although this method is quite promising and has a good resolution, it can only give a discrete map of traction forces, as opposed to the classical method of Dembo and Wang [9] which, in principle, provides the value of traction forces at any point.

1.2 The method of Dembo and Wang (1999) and recent improvements

\subsubsection{Description}

We assume that the polyacrylamide substrate (on which cells are deposited) is elastic when observed at a time scale of the order of minutes to hours; this means that such a material may actually be viscoelastic, but relaxation times are much larger than the observation time. Under assumptions of isotropy (no preferential directions) and homogeneity (no explicit dependence on space), the deformations are supposed to be small: in a quantitative sense, this means that

$$
\operatorname{trace}\left((\nabla \mathbf{u})^{T} \nabla \mathbf{u}\right) \ll 1,
$$

where $\mathbf{u}(x, y, z)=(u, v, w)$ is the displacement field. Neglecting body forces and inertia, the balance equations for the substrate read

$$
-\nabla \cdot \mathbf{T}=0
$$

where

$$
\mathbf{T}=\mu\left(\nabla \mathbf{u}+(\nabla \mathbf{u})^{T}\right)+\lambda \nabla \cdot \nabla \mathbf{u}
$$

is the Cauchy stress tensor and $\mu, \lambda$ are the Lamé coefficients. The following boundary conditions apply:

$$
\begin{gathered}
\operatorname{Tn}=\mathbf{f}(x, y), \quad z=0 \\
\mathbf{u} \rightarrow 0, \quad z \rightarrow-\infty
\end{gathered}
$$

where $\mathbf{n}$ is the vector pointing in the $z$ direction (vertical) and $\mathbf{f}$ is the traction exerted by the cell at the surface. If the displacement of the substrate is known at some points on the surface, say $\mathbf{u}_{o}$ its value, it is quite obvious that we cannot plug this directly into (1.2) to obtain $\mathbf{f}$. The motivations are twofold: since $\mathbf{u}$ is constrained to equal $\mathbf{u}_{o}$ in some portions of the domain only, there are many $\mathbf{f}$ that can produce this known displacement. Secondly, inverse problems are well known to excite high frequency components of the (always present) experimental error and a regularization procedure is therefore needed [32]. 
Simple dimensional arguments can show that the substrate displacement is non-negligible within heights of a few microns [32]. Since substrates are typically one hundred microns in height, the approximation of infinitely deep half space applies and a relatively simple Green function provides the solution of the elasticity problem. For thinner and finite substrates, there is a much more intricate Green formulation [23]. The solution of the problem (1.2-1.4) can then be rewritten in integral form using the Green tensor $\mathbf{G}$ of the elasticity equation for the half space domain [19]:

$$
\mathbf{u}(\mathbf{x})=\int \mathbf{G}\left(\mathbf{x}-\mathbf{x}^{\prime}\right) \mathbf{f}\left(\mathbf{x}^{\prime}\right) d \mathbf{x}^{\prime}
$$

where the integration domain is the support of $\mathbf{f}$, that is the area covered by the cell. If any information about the focal adhesion points is available, they can be used at this stage. In practice, for every non-trivial $\mathbf{f}$ the integral (1.5) has to be evaluated numerically. Three assumptions are now commonly adopted before solving the problem numerically:

1. The substrate material is incompressible.

2. The cell exerts shear stress only, so that $\mathbf{f}=\left(f_{x}, f_{y}, 0\right)$.

3. The measured displacement $\mathbf{u}_{o}$ corresponds to beads located at the very surface of the matrigel. From a practical point of view, the focus length of the experimental pictures must be much smaller than the characteristic vertical length of decay of the tensional field. As the latter is of the order of few microns, both beads radius and focus length should be order of a micron at most.

If assumptions 1 and 2 apply, the vertical component of the displacement at the surface is identically zero and the Green tensor takes the following simplified form [32]

$$
G_{i j}=\frac{3}{4 \pi E r}\left(\delta_{i j}+\frac{x_{i} x_{j}}{r^{2}}\right),
$$

where $x_{1}=x, x_{2}=y, r^{2}=x^{2}+y^{2}$. In terms of the Lamé coefficients, the Young modulus $E$ is defined by

$$
E=\frac{\mu(3 \lambda+2 \mu)}{\lambda+\mu}
$$

The Green tensor allows one to calculate the surface displacement by the following simplified version of the convolution (1.5)

$$
u_{i}(x, y)=\int G_{i j}\left(x-x^{\prime}, y-y^{\prime}\right) f_{j}\left(x^{\prime}, y^{\prime}\right) d x^{\prime} d y^{\prime}
$$

Formula (1.8) provides the horizontal displacement at $z=0$ given a pure shear stress $f_{i}$. If the beads are sufficiently small and shallow, assumption 3 applies and the computed displacement field $u_{j}$ can be compared with the measured one.

The target of this methodology is to find the force per unit surface $f_{i}$ generating a displacement very near to the experimental one in a suitable sense. The usual approach is to minimize the quadratic mean error under force penalization [9] [32] to ensure regularization. The basic idea of the Tikhonov regularization method is also used in the next section in a different framework; therefore no details are provided herein and the reader is referred to the cited literature for details. In this context we just remark that the error minimization procedure is decoupled from the mechanical one and applies to the discrete problem obtained covering the cell area by polygons (triangles or quadrilaterals) where the numerical integration (1.8) is carried out. 


\subsubsection{Improvements}

The Fourier Transform Traction Cytometry (FTTC) method used by Butler and coworkers [7] is based on the observation that equation (1.8) can be conveniently solved in the Fourier components space, taking advantage of the properties of the convolution product. A simple linear relationship between the displacements and the forces in the Fourier space is obtained. The method has been used successfully to compute the motion of smooth muscle cells on elastic substrates [7].

Recently, the use of thinner substrates [23] has been proposed and it seems to give rise to accurate results, due to improved spatial resolution. This has been made possible thanks to the use of the Green function for finite thickness elastic layer.

Finally, a recent paper [31] came to our attention recently where the authors compared the efficiency and accuracy of the methods above (Boundary Element Method BEM [9], Fourier Traction Force Cytometry FTTC [7], and Traction Reconstruction with Point Forces [32]). It was shown that the first two methods can be improved to reach spatial resolutions of $1 \mu \mathrm{m}$, and combined with the third one can lead to new advances in cell mechanics understanding.

\section{Force balance and adjoint equation}

In this section we briefly describe an alternative approach to obtain the pattern of the shear stress exerted by the cell: the adjoint method [2]. If $\Omega$ is the half space, the displacement vector field $\mathbf{u}(\mathbf{x})$ is known in a subset of the domain of the elasticity equation $\Omega_{0} \subset \Omega$, where beads are located. The target function $\mathbf{u}_{0}(\mathbf{x})$ has support in $\Omega_{0}$. In this problem the shear stress is exerted just on the portion of the domain where the cell lies; let us call this subdomain $\Omega_{c} \subset \Omega$ (see figure 2). The cell actually adheres to the substrate just in specific small regions called focal adhesion sites, which can be experimentally localized [1] using fluorescence for instance. No reason prevents restricting the force support to these areas and, as a matter of fact, this information is included in refinements of the algorithm of Dembo and Wang [31]. This assumption is not applied here just because the information is missing from the experiments.

Here the three-dimensional elasticity system of equations is approximated by a twodimensional plane-stress one by vertical averaging along an effective thickness $h$ :

$$
-\hat{\mu} \Delta \mathbf{u}-(\hat{\mu}+\hat{\lambda}) \nabla(\nabla \cdot \mathbf{u})=\mathbf{f},\left.\quad \mathbf{u}\right|_{\partial \Omega}=0
$$

where

$$
\hat{\mu}=h \frac{E}{2(1+\nu)}, \quad \hat{\lambda}=h \frac{E \nu}{1-\nu^{2}} .
$$

and $E$ and $\nu$ are the Young modulus and the Poisson ratio respectively. $h$ is the averaging depth fixed by the depth of field of the microscope. In our case $h$ is 1.5 microns; the beads lying below such vertical coordinate are not in focus and therefore their position is not measured. Consequently the displacement $\mathbf{u}$ should be understood as the average displacement along $h$, which is nearly the displacement of the center of the beads.

The functional $J(\mathbf{f})$ measures the difference between the displacement field produced by $\mathbf{f}$ and the experimental one $\mathbf{u}_{0}$ under penalization of the square norm of the force 


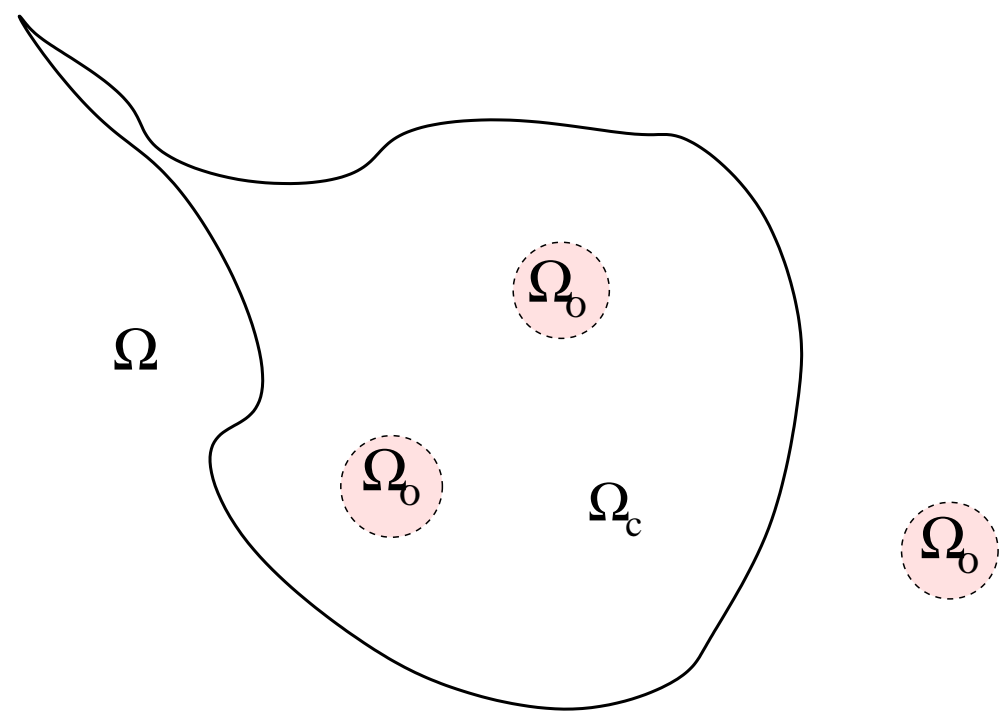

Fig. 2 The domain $\Omega$ of the elasticity equation contains the subdomain $\Omega_{c}$, the area covered by the cell, where the force applies: in the figure it is enclosed by the continuous bold line. The dashed circles are centered at the beads location and their collection represents the $\Omega_{0}$ subdomain where the displacement is known.

field itself. It is defined as follows:

$$
J(\mathbf{f})=\int_{\Omega_{0}}\left|\mathbf{u}-\mathbf{u}_{0}\right|^{2} d V+\varepsilon \int_{\Omega}|\mathbf{f}|^{2} d V,
$$

where $\varepsilon$ is a real positive number. We look for $\mathbf{g}$ minimizing $J$ :

$$
J(\mathbf{g}) \leq J(\mathbf{f}), \quad \forall \mathbf{f} \in V_{c}
$$

where $V_{c} \subset L^{2}(\Omega)$ is the space of the finite energy functions with support in $\Omega_{c}$. The minimization of $J$ accomplishes the minimization of the distance of the solution from the measured value $\mathbf{u}_{0}$ under penalization of the magnitude of the associated force per unit surface $\mathbf{f}$. The penalty parameter $\varepsilon$ balances the two requirements.

Variational derivation of $J(\mathbf{f})$ and introduction of the adjoint differential equation yields the following direct and inverse systems of partial differential equations [2]

$$
\begin{aligned}
& -\hat{\mu} \Delta \mathbf{u}-(\hat{\mu}+\hat{\lambda}) \nabla(\nabla \cdot \mathbf{u})=-\frac{\chi_{c}}{\varepsilon} \mathbf{p},\left.\quad \mathbf{u}\right|_{\partial \Omega}=0, \\
& -\hat{\mu} \Delta \mathbf{p}-(\hat{\mu}+\hat{\lambda}) \nabla(\nabla \cdot \mathbf{p})=\chi_{o} \mathbf{u}-\mathbf{u}_{0},\left.\quad \mathbf{p}\right|_{\partial \Omega}=0 .
\end{aligned}
$$

The value of the penalty parameter $\varepsilon$ and the averaging depth $h$ can be fixed on the basis of arguments suggested by modal analysis. In the special case $\Omega_{0}=\Omega_{c}=\Omega$ under periodic boundary conditions, modal analysis applies and the system of Equations (2.4) rewrites just like a Tikhonov filter. The amplitude of the Fourier components of the 
solution $u_{k}, p_{k}$ satisfies the algebraic relation

$$
\begin{aligned}
h E k^{2} u_{k} & \simeq-\frac{1}{\varepsilon} p_{k}, \\
h E k^{2} p_{k} & \simeq u_{k}-u_{0, k},
\end{aligned}
$$

that is

$$
u_{k} \simeq \frac{u_{0, k}}{1+\varepsilon h^{2} E^{2} k^{4}}
$$

where $u_{0, k}$ represents the amplitude of the $k$-th Fourier component of $u_{0}$. According to equation (2.6), if the data is known all over the domain the system of equations (2.4) is nothing but a filter damping the modes corresponding to wavenumbers $\gtrsim \varepsilon^{-1 / 4} h^{-1 / 2} E^{-1 / 2}$. The choice of $\epsilon$ can be interpreted in terms of filtering modes falling below the experimental accuracy. A closer inspection of Equation (2.6) reveals that the key parameter of the inversion procedure is actually $h^{2} \epsilon$ and the solution does not change for combinations of the averaging layer $h$ and penalty parameter $\epsilon$ that preserve this quantity.

The choice of the penalty parameter (also called regularization parameter in discrete inverse problems) is a delicate subject and it has been extensively discussed in the relevant literature. Basically it should be chosen to damp components whose wavenumber has no physical meaning because they are below the experimental resolution. It is evident that no inversion technique can account for variations of the shear stress at a spatial scale smaller than the minimum distance between two beads and, even though the beads are quite dense, the determination of the position of their centers is subject to a noise. Several possible strategies can be addressed to find out the optimal value of $\epsilon$ in a suitable way; the interested reader can refer to the very accurate paper by Schwarz et al. [32]. In this work we simply take the minimum value of $\epsilon$ that does not yield erratic results in the displacement (the L-curve criterion).

\section{Materials and methods}

In this paper the mathematical methodology illustrated above is applied to determine the stress field exerted by T24 tumor cells on a flat deformable substrate. The experimental procedures that have been used are based on the work by Dembo and Wang [9] and are given below.

- Gels of different stiffness have been prepared by tuning the ratio between polyacrylamide and bis-acrylamide components. Three different gels have been used containing $x \%$ of polyacrylamide ( $x$ taking the values $5-7.5-10$ going from the softer to the harder gel) and the bis-acrylamide percentage is $0.03 \%$. Their mechanical properties have been measured by conventional dynamic rheometry tests (Malvern rheometer, Gemini 150). Sinusoidal oscillations with a known deformation $\gamma=\gamma_{0} \sin (\omega t)$ are applied within the linear regime (small enough deformation $\left.\gamma_{0} \sim 0.01\right)$ at different angular frequencies $\omega$. The stress response $\sigma=\sigma_{0} \sin (\omega t+\phi)$ (where $\sigma_{0}$ is a constant stress and $\phi$ is the phase angle) is measured and the elastic $\left(G^{\prime}\right)$ and viscous moduli $\left(G^{\prime \prime}\right)$ are deduced.

Experiments show a constant $G^{\prime}$ (elastic modulus) when the frequency $\omega$ ranges from 0.1 to $10 \mathrm{~Hz}$. The loss modulus $G$ " is usually lower by two orders of magnitude (data not shown). We deduce the value of the elastic modulus $E=3 G^{\prime}$ and find 
$1.95 \mathrm{kPa}, 6.3 \mathrm{kPa}$ and $9.9 \mathrm{kPa}$ for the soft, medium and hard gels respectively. Note that the hypothesis that $E=3 G^{\prime}$ is relevant here in view of a recent work [4] showing that $\nu \sim 0.48$ in such polyacrylamide gels. This means that our hypothesis of incompressible material (i.e. $\nu=0.5$ ) is quite good, and is not responsible for the differences found as compared to other methods. Such comparisons are shown in Figure 3 where our results are found to be close to the ones obtained by Pelham and Wang [25] or Boudou et al. [4]. Since our method relies on no further hypotheses and is based on the use of large samples, we have good confidence in our data. Other techniques which can be used are traction tests [13,25], micropipette experiments [4], AFM [13,14], or rheometry [35].

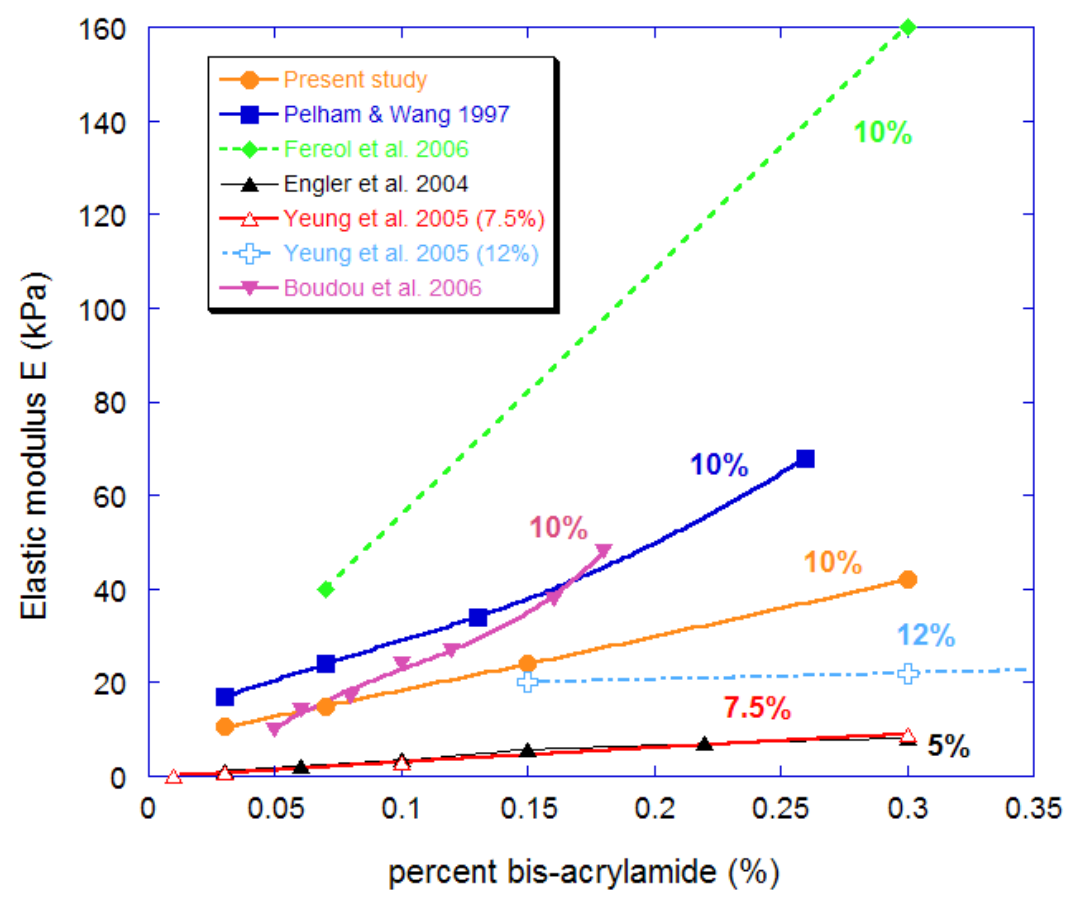

Fig. 3 Elastic moduli $\mathrm{E}(\mathrm{kPa})$ as a function of the bis-acrylamyde percent. Values from other authors are also reported $[4,13,14,25,35]$ for the case of $10 \%$ polyacrylamide concentration and a few other concentrations.

Gels were prepared on a silanated square coverglass $22 \mathrm{~mm} \times 22 \mathrm{~mm}$ and covered with a circular coverglass $(35 \mathrm{~mm}$ diameter) functionalized with $\mathrm{NaOH}(0.1 \mathrm{M})$, APTMS (10mn), and $0.5 \%$ glutaraldehyde $(30 m n)$.

- Fluorescent beads (Molecular Probes) of 0.2 micrometers of radius were seeded as the gels were prepared. After addition of the cross-linker, beads were added, the gels were set onto the square coverglass, and the circular coverglass was brought carefully to capture the gel and the square coverglass. This avoided to flip the preparation. Indeed beads need to sediment fast so that there will come close to the gel upper surface. 
- After the gel was polymerized (nearly 30 minutes), the square coverglass was removed and sulfo-Sanpah $1 \mathrm{mM}$ was added to functionalize the gel (15 minutes under UV). This was achieved twice, and the surface was rinced with PBS. Finally a $20 \mu \mathrm{g} / \mathrm{ml}$ fibronectin solution was used overnight to bind the above surface.

- Cancer cells of epithelial bladder type (T24) were then seeded. They adhered usually rapidly and spred. This cell line is known to be of an average invasive type.

- The coverglass was attached at the bottom of a $35 \mathrm{~mm}-$ culture dish (containing culture medium) in order to carry out microscopic observations. Two types of images were made: a phase contrast one to observe the cell and its contour, and a fluorescent one focused on the beads (at a slightly different $\mathrm{z}$-position). The depth of field of the images was 1.5 micrometers. Everything was carried out automatically in order to take one set of images at regular time steps (10mn, for example).

- Images were then collected and treated using the ImageJ software [18], to determine trajectories and/or displacements with respect to the initial position. The initial beads position was determined at the end of the experiment by adding distilled water to detach the cells.

\section{Numerical results}

Equations (2.4) have been discretized by a finite element method using linear basis functions on an unstructured mesh. The two resulting linear systems were solved numerically using a global conjugate gradient method, thus avoiding any iterative coupling [28]. The computational domain was a square box with side of about 100 microns. The Young moduli of the substrates are $9.9 \mathrm{kPa}, 6.3 \mathrm{kPa}, 1.95 \mathrm{kPa}$, as detailed in the previous section.

In Figure 4 an example of the numerical setup is shown: in a part of the domain, the cell contour, the displacement of the beads and the computational mesh are plotted. The cell contour represents the boundary between internal and external elements. Note that some nodes of the mesh correspond to the original beads location while others do not: they have been created for the sake of regularity of the computational grid. The present approach ensures a full flexibility in this respect. According to the notations introduced in the previous sections, the cell contour defines $\Omega_{c}$ while the collection of the elements that have at least one node with measured displacement defines $\Omega_{0}$.

The computed displacement $\mathbf{u}$ is in general different from $\mathbf{u}_{o}$, the difference increasing for larger $\varepsilon$. The mean difference between the calculated and the measured solution for the specific case of Figure 4 is

$$
\frac{1}{n} \sqrt{\sum_{i=1}^{n}\left(\mathbf{u}_{i}-\mathbf{u}_{0, i}\right)^{2}}=5.310^{-3} \mu \mathrm{m}
$$

where the sum runs over all the nodes where $\mathbf{u}_{o}$ is known. The mean quadratic error has this order of magnitude for all the computations to be shown below.

In Figures 5-7 cell pictures are shown together with the numerical results corresponding to gels showing decreasing stiffnesses. The first image is (a) the phase-contrast image of the cell; note that in this representation the beads are not visible: as they fluoresce they are recorded with the fluorescent microscopy technique. Beads displacements are shown in (b) after particle tracking is performed thanks to the ImageJ software. The shear stress is shown in terms of vectors (c) or color map showing the magnitude (d). 


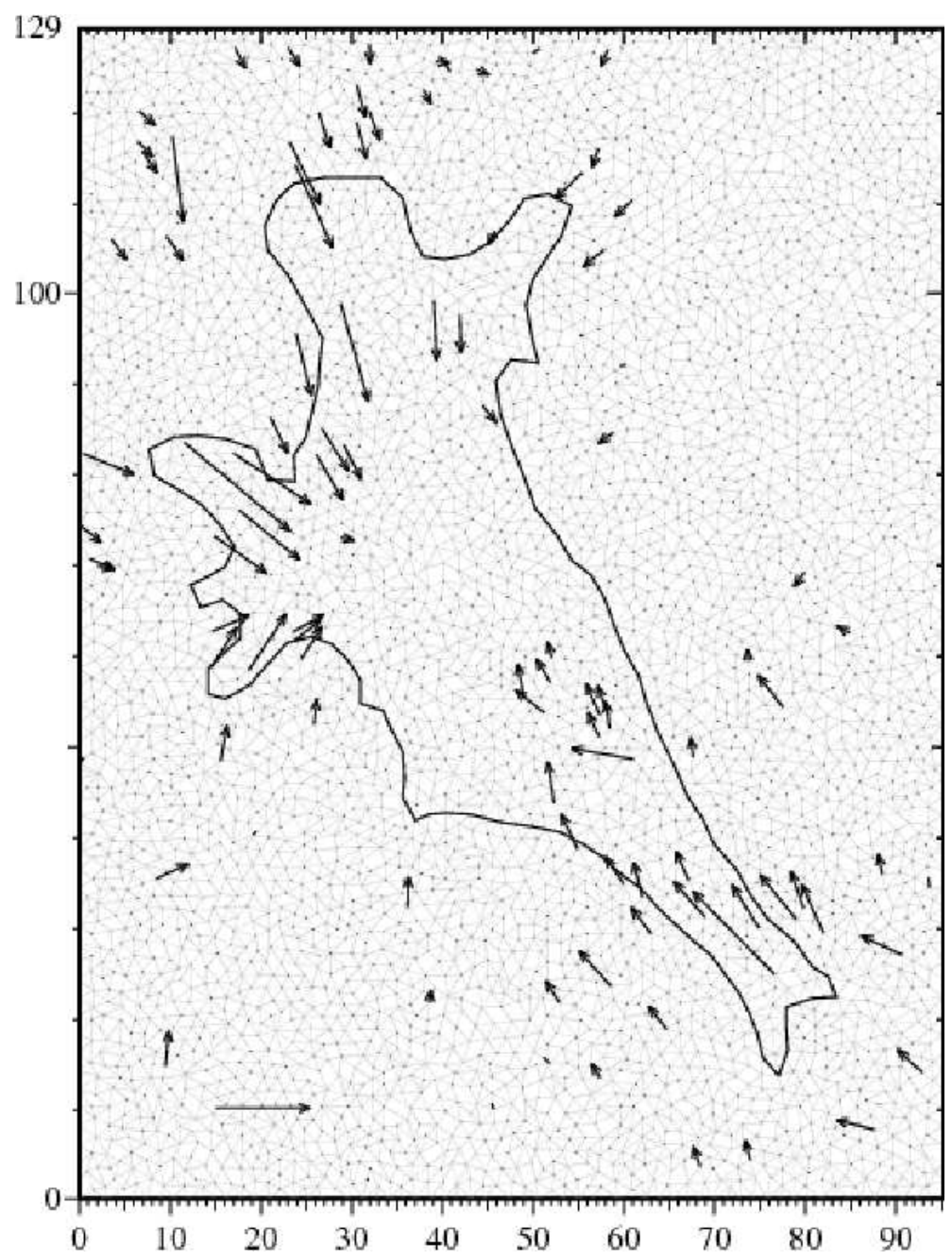

Fig. 4 Graphical representation of the numerical setup: the computational mesh, made of triangles, is represented in light grey. The mesh satisfies two constraints: it has a node at every point where displacement is known (the arrows have been measured and a sequence of element sides coincides with the boundary of the cell. The reference vector at the bottom left corner is 0.5 microns long.

The experimental and numerical results show some features that are well known in the relevant literature and here read as a confirmation of the validity of the procedures. Cells are more convex and more active when adhering to a soft substrate, whereas they are more elongated and develop larger forces on a stiff substrate. Secondly, the force per unit surface generated by tumor cells $\left(\sim 100 \mathrm{pN} / \mu \mathrm{m}^{2}\right)$ is weaker than the one typically exerted by fibroblasts, which is of the order of thousands of picoNewton per micron squares, and have been originally used in the literature to apply this kind of methodology $[9,22]$. 

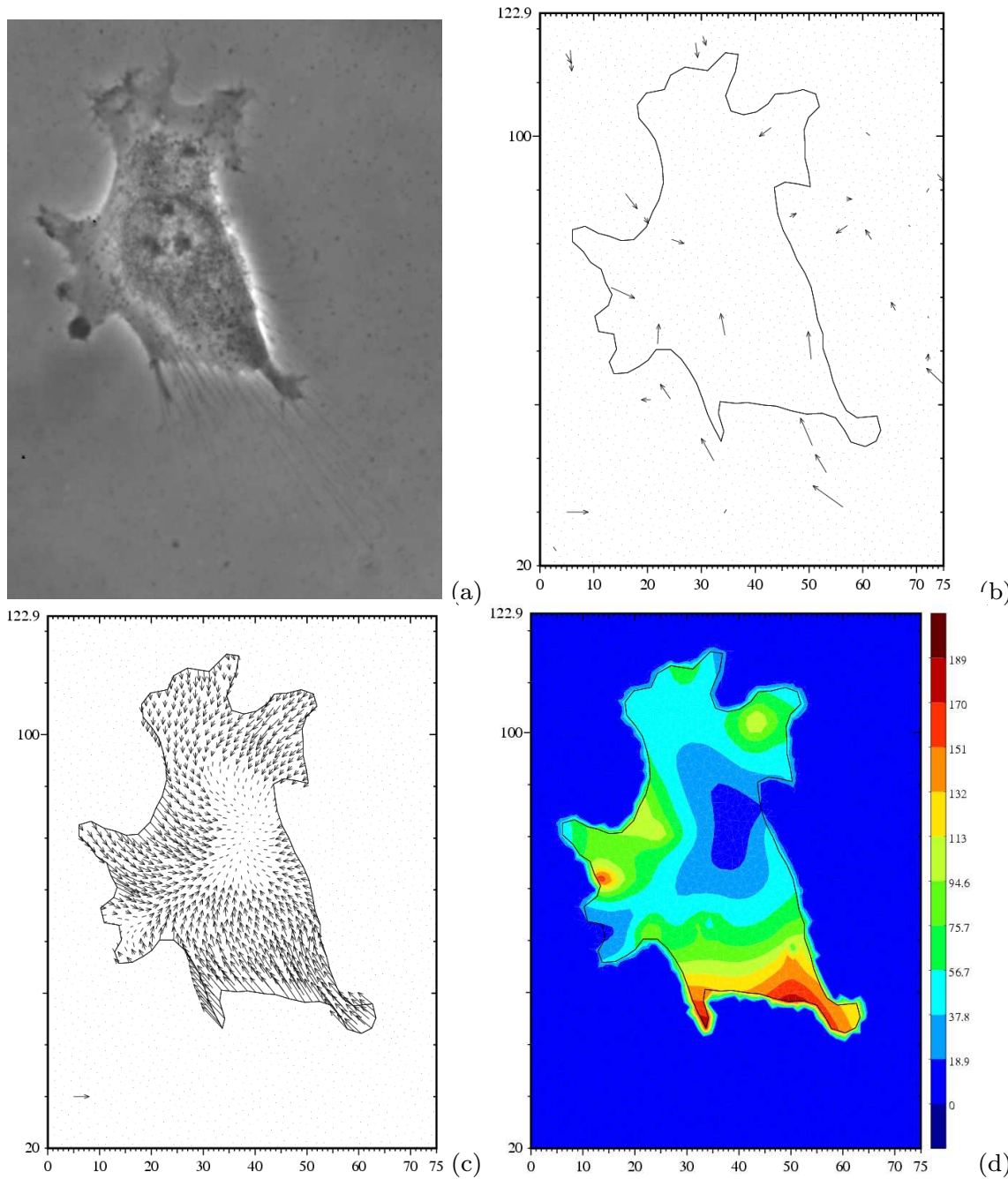

Fig. 5 T24 cell adhering on a stiff polyacrylamide substrate $(E=9.9 \mathrm{kPa})$. The cell is quite flattened on the surface and exhibits a spiky contour (a). Some displacement vectors below and around the cell are known (b). The axis scale is in microns. The shear stress is shown in terms of vectors (c) or color map of the magnitude (d). The traction force has maximum magnitude corresponding to about $200 \mathrm{pN} / \mu \mathrm{m}^{2}$. Note that on this substrate the cell produces filopodia which appear on the edges and attach the gel out of the cell contour. Filopodia seem to have a minor dynamical effect being essentially aimed at addressing the direction of the motion and their role is not taken into account in the present model. Reference vectors for displacement and stress stand for $0.5 \mu \mathrm{m}$ and $100 \mathrm{pN} / \mu \mathrm{m}^{2}$ respectively.

The migration of T24-cells on the most rigid substrate, as an example of random migration for such invasive cells, is shown in Figure 8. The T24-cell is first adhering in the lower right part. Then it begins to move in a random manner until it elongates a bit then it starts to move its upper right part to each side to see whether it can bind efficiently. This is achieved after roughly $16 \mathrm{mn}$ and new adhesions are formed in the 

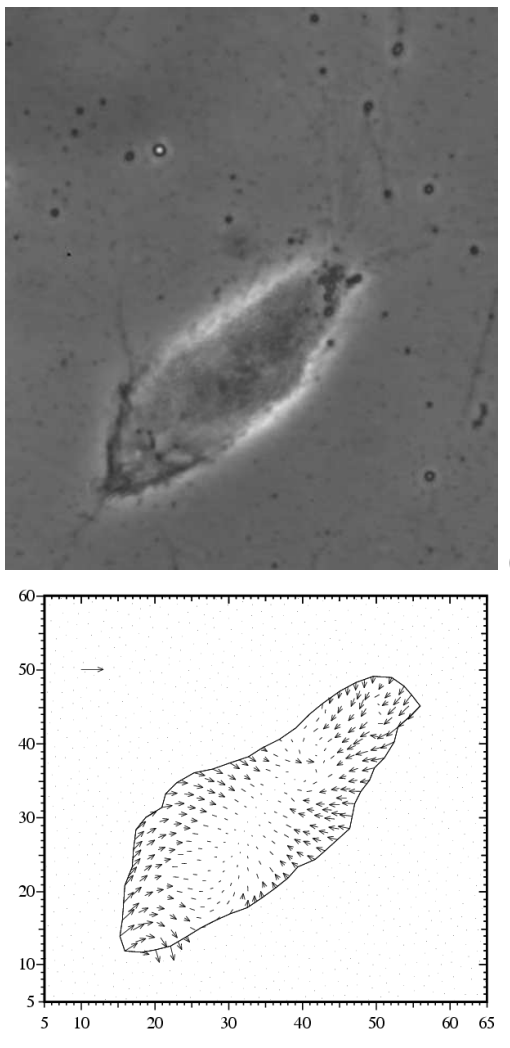

(a)

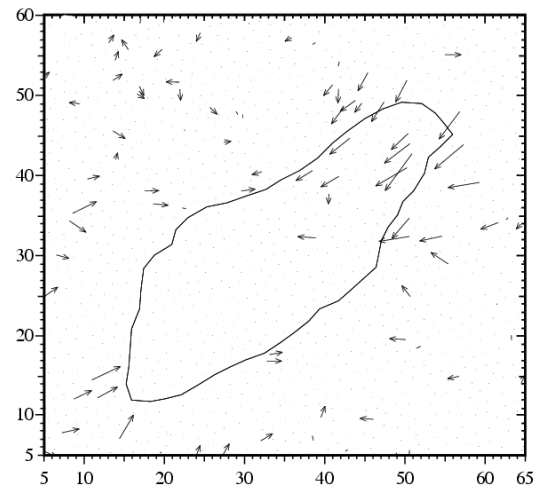

(b)

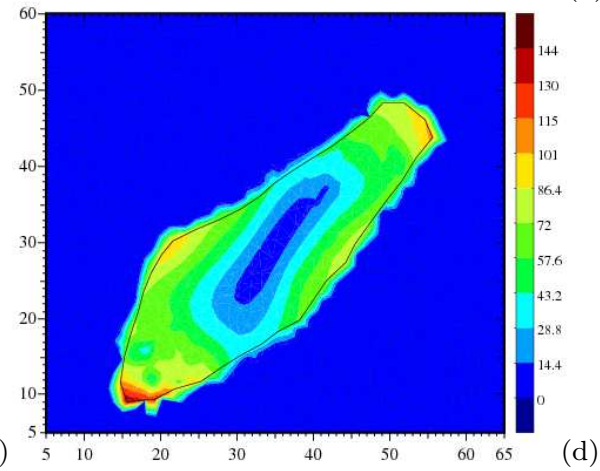

Fig. 6 T24 cell adhering on a medium stiffness polyacrylamide substrate $(E=6.3 \mathrm{kPa})$. The cell is more rounded than in the rigid case (a) while the displacement field appears to be of the same order of magnitude (b). The axis scale is in microns. The shear stress is shown in terms of vectors (c) or color map of the magnitude (d). The traction force has maximum magnitude corresponding to about $140 \mathrm{pN} / \mu^{2}$. Note that in this case few beads are detected in focus under the cell and consequently the stress field is less reliable. Reference vectors for displacement and stress stand for $0.5 \mu \mathrm{m}$ and $100 \mathrm{pN} / \mu \mathrm{m}^{2}$ respectively.

upper left part. This can be seen by the larger forces in red in Figure 8d-e. At the same time, the high forces produced make it break its lower right adhesion site (in red also) as seen in Figure 8e-f. This large adhesion site is removed and the cell contracts its rear part to join the rest of the cell (upper right).

\section{Discussion}

\subsection{Modelling aspects}

In this paper an adjoint-based method has been applied for solving the inverse problem to obtain the shear stress exerted by T24 tumor cells on an elastic substrate. The novelty of the paper is the application of a recent methodology (alternative to Dembo and Wang) to determine the stress exerted by a particular cell line (T24 tumor cells) not yet investigated. 

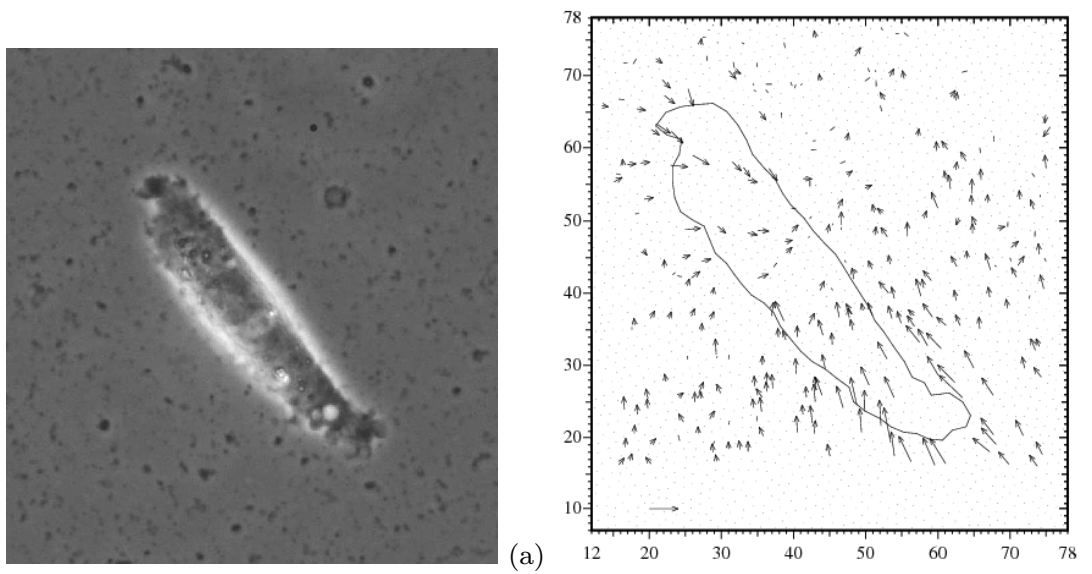

(b)
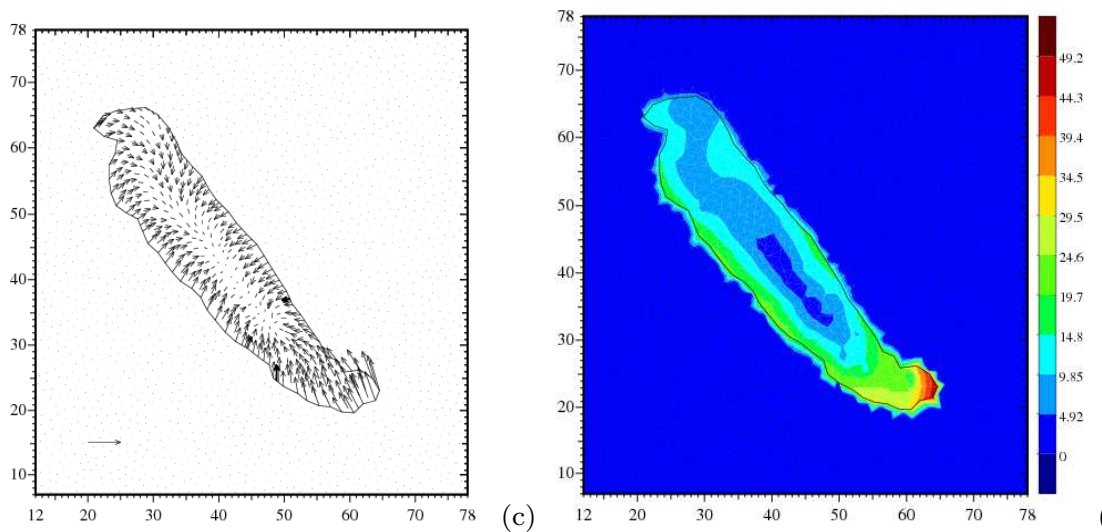

(d)

Fig. 7 T24 cell adhering on a very soft polyacrylamide substrate $(E=1.95 \mathrm{kPa})$. The cell is more convex than in the rigid case (a) while the displacement field appears to be of the same magnitude (b). The axis scale is in microns. The shear stress is shown in terms of vectors (c) or color map of the magnitude (d). The traction force has maximum magnitude corresponding to nearly $50 \mathrm{pN} / \mu^{2}$. Reference vectors for displacement and stress stand for $0.5 \mu \mathrm{m}$ and $100 \mathrm{pN} / \mu \mathrm{m}^{2}$ respectively.

A few comments can be drawn on a theoretical basis. The classical method of Dembo and Wang is based on the knowledge of the exact solution of the elasticity equation in a half plane under linearity assumptions, and for an isotropic and homogeneous medium. By numerical quadrature such an exact solution is part of a discrete minimization algorithm that provides the shear stress under regularization based on the Tikhonov method.

Conversely, the adjoint method does not exploit the knowledge of an exact solution and does not decouple the direct and inverse problems: variational arguments yield two coupled sets of partial differential equations to be solved by a suitable numerical method (Finite Elements, for instance).

The computational cost of the two methods can be estimated for a shear force f to be calculated at $N$ points. The method by Dembo and Wang requires $N$ sums to compute the integral (1.8) for all the $N$ nodes, while the solution of the linear 

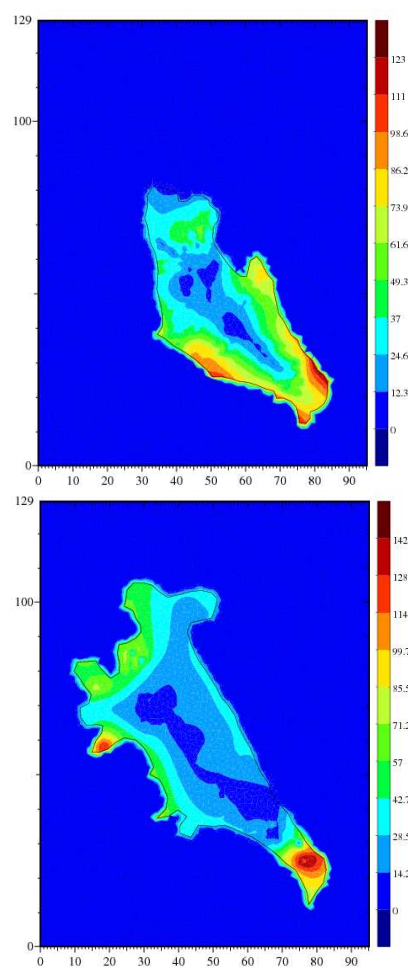

(a)
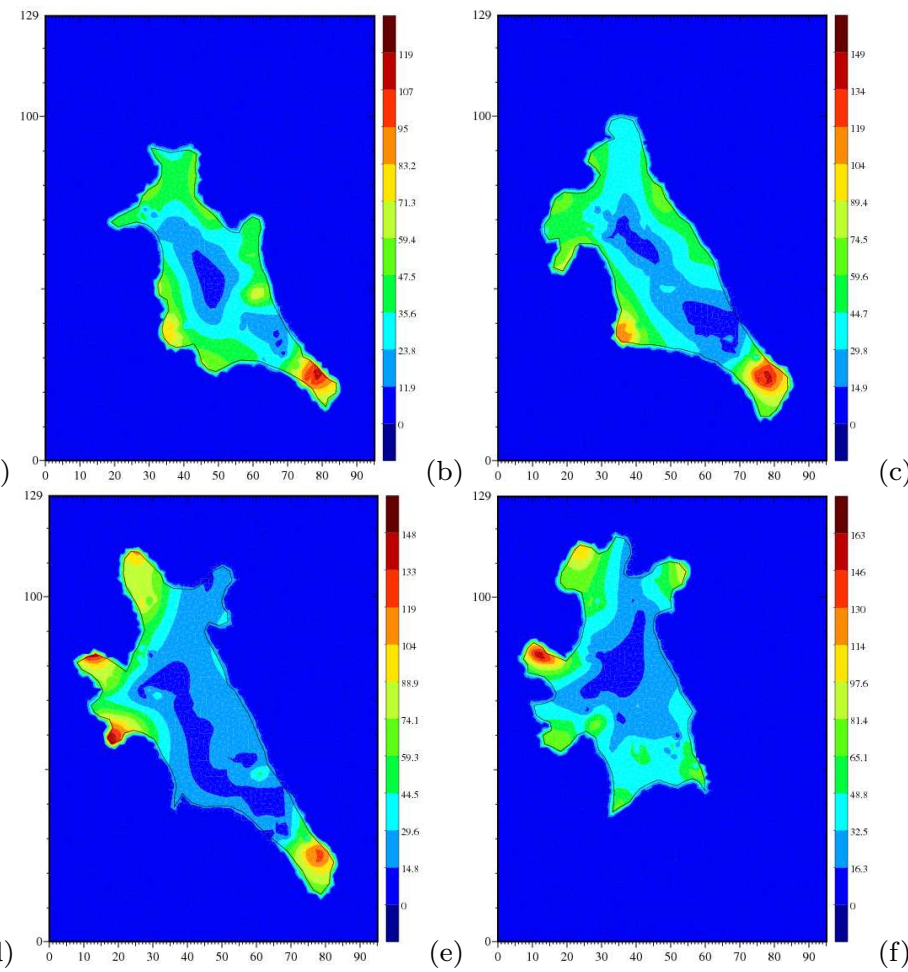

(c)

Fig. 8 Motion of a T24 cell on a rigid gel $(E=9.9 k P a), t=0,8,16,24,30,40 \mathrm{mn}$. The cell first adheres strongly (red region) at its lower right part (a), then starts to move upward left by random migration (b-c-d) until it eventually forms new adhesion sites at the upper left sites (d-e). At this precise time, it is able to contract and detach its rear by first decreasing forces while elongating (e), then achieving detachment to bring the rest of the body to the upper left part (f). Note that the colour scale is reset to range between minimum and maximum in each frame.

system arising from the finite element discretization is usually solved by an iterative linear solver that typically involves order $N$ operations. Therefore the computational cost of the adjoint method scales like $N$, while the usual one scales like $N^{2}$. This difference is essentially due to the local nature of the finite element basis, leading to a sparse stiffness matrix. Conversely, the quadrature (1.8) is an (explicit) sum spanning the whole computational domain. This issue has been addressed by Sabass et al. [31] who proposed a splitting of the elastic field into spatial ranges that require a different numerical accuracy.

The adjoint method is approximate because it does not use an exact solution of the elasticity equation, but a vertically averaged system of equations between 0 and $-h$. However, the non-dimensional number characterizing the differential equations involves this somehow arbitrary vertical height through a combination of $h$ and $\epsilon$, which is an actual parameter to be fixed by the regularization method. Both methods require numerical integration. A specific character of the adjoint method is that it automatically satisfies the force equilibrium condition: integrating equation (2.4) over a domain containing $\Omega$, making use of the divergence theorem, then one immediately finds that the average force $\mathbf{f}$ is zero, as expected for a system in equilibrium. 


\subsection{Experimental results}

In order to compare our results with previously published works, we first looked at the results by Dembo and Wang [9] and compared them with Lo et al. [22] where migrating 3T3 fibroblasts on collagen-coated gels are studied. For the $6 k P a$ polyacrylamide gel, maximum traction forces on the edges are about $7 k P a$ [9]. In the second paper [22], respective values of $6 \mathrm{kPa}$ and $11 \mathrm{kPa}$ are found for the forces exerted on gels of rigidity $14 k P a$ and $30 k P a$ respectively, and velocities are roughly $0.4 \mu \mathrm{m} / \mathrm{mn}$ and $0.2 \mu \mathrm{m} / \mathrm{mn}$. This means that lower values are found. Finally, in the recent work of Sabass et al. [31], mouse embryo fibroblasts are shown to develop maximum traction forces of $2 \mathrm{kPa}$ on a $10 \mathrm{kPa}$ polyacrylamide substrate. These values are more or less in the same range and give an order of magnitude, although they do not seem to be reproducible. Another approach uses epithelial Madin-Darby canine kidney cells (MDCK) on an array of microneedles [12]. It is a bit difficult to compare the data because the matrix rigidity is not exactly determined (the crosslinked silicone substrate making the microneedles is known to have an elastic modulus of $1.5 \mathrm{MPa}$, but nothing is said about the whole equivalent substrate). On such a substrate, MDCK cells exert stresses of $1 k P a$, a value similar to the ones developed by fibroblasts. Our results on T24 cell migration clearly show much smaller values of maximum forces, in the range $0.05-0.2 k P a$. This is a new and promising result, suggesting possible applications of this study to cancer cell migration in general. Other comparisons can be made with endothelial cells [26] moving on RGD-coated polyacrylamide gels $(2.5 \mathrm{kPa})$ with traction forces in the range of $2-8 k P a$. To our knowledge, the only case where such small forces are found is that of airway smooth muscle cells (HASM) advancing on collagen-coated polyacrylamide gels $(E=1.2 k P a)[7]$ exhibiting traction forces in the range of $0.1-0.4 k P a$. The precise mechanisms to explain such behaviors still need to be understood.

Durotaxis has been studied previously and reveals the ability of cells to develop large focal adhesions when in contact with a rigid substrate. This type of mechanism, discussed by Choquet et al. [8] is dependent on the growth of contact adhesions mainly of the integrin-cytoskeleton type. In particular, it was shown [22] that traction forces are stronger when the matrix rigidity increases. This work is another confirmation of this result because, as shown in Table 1, the maximum force exerted by T24 cells increases (from $0.05 k P a$ to $0.2 k P a$ ) with the elastic gel modulus (from $1.95 k P a$ to $9.9 k P a)$. Although this result is not new, it shows that this cancer cell line behaves in a similar manner on an elastic gel. In another approach, Saez et al. [29] have shown that MDCK cells on a 'fakir' substrate with different needles rigidity exert traction forces proportional to the elastic spring constant of the needles. This would mean that the ratio of force to elasticity is constant, in other words the deformations are the same whatever the rigidity. This is also what was postulated by Discher et al. who made the same observations [11] and found that typical strains on such deformable substrates come close to $3-4 \%$. As shown in table 1 , it seems that such an assumption is not so crude, because our findings come close to a constant ratio of the parameter $\frac{\text { Maxstress }}{\text { Rigidity }}$, of the order $2 \%$ within experimental uncertainty.

In Figure 8, we exhibited for the first time the motion pattern of a whole cell in terms of traction forces. This way of locomotion is similar for several types of cells. Only keratocytes $[6,20]$ have the ability to move with a 'crescent' shape by pulling mainly on the sides. The way T24 cells move is more standard and comparable to the classical four-step picture [1] which requires the formation of a lamellipodium at the front, the development of new focal adhesions, the contraction of the cell, and the 
Table 1 Features of T24 cells on different gels.

\begin{tabular}{llll}
\hline $\begin{array}{l}\text { Gel rigidity } \\
(\mathrm{kPa})\end{array}$ & $\begin{array}{l}\text { Max. stress } \\
(\mathrm{kPa})\end{array}$ & $\begin{array}{l}\text { Velocity of migration } \\
(\mu \mathrm{m} / \mathrm{mn})\end{array}$ & $\begin{array}{l}\text { Stress/Rigidity } \\
(\text { adimensional) }\end{array}$ \\
\hline & & & \\
1.95 & 0.05 & 1.2 & 0.026 \\
6.3 & 0.14 & 0.4 & 0.022 \\
9.9 & 0.2 & 0.2 & 0.02 \\
\hline
\end{tabular}

release of bonds at the rear. Figure 8 illustrates these mechanisms perfectly in terms of forces. Our T24 cell first explores new regions until it binds, then it pulls on these bonds to detach the rear part (uropod). We have determined the velocity of migration of such a motion; it is presented in Table 1 . We can clearly see that the cell velocity is larger on less rigid gels. This is in agreement with other works [11,22], and this idea is explained by the ability of a less-adhering cells to move faster, as they do not require to detach strong bonds. Finally, we may conclude that cell migration is a very complex mechanism which requires to take into account several aspects: cell adhesion/substrate affinity, cell microrheology [34] i.e. its ability to change its mechanical properties, and cell signaling as well as biochemical activity. A clear example of this complexity is given by the bell shape of the migration velocity curve as a function of substrate ligand density [24]. We do not pretend to give an answer to this difficult mechanism, but the simple resolution proposed here already retains the major common aspects of cell migration.

The present method has been applied to study the traction ability of T24 cancer cells adhering to a polyacrylamide substrate of tuned stiffness, with Young moduli ranging roughly from $2 \mathrm{kPa}$ to $10 \mathrm{kPa}$. Further statistical analysis are still required to investigate more results. Although this technique may not be as accurate as recently proposed ones [23,31], it still allows to confirm features already observed with other cells (influence of substrate rigidity, forces, velocities), as shown here. It may become a very valuable tool to quickly study the dynamics of migrating cancer cells, in relation with their invasiveness. In addition, other aspects of cell properties (interactions, collective effects, time-dependent processes) might also be studied efficiently.

\section{Acknowledgments}

The authors are indebted to Luigi Preziosi for fruitful discussions about the content of this paper. This research was partially supported by the EU Marie Curie Research Training Network MRTN-CT-2004-503661 "Modelling, Mathematical Methods and Computer Simulation of Tumour Growth and Therapy".

Image acquisition was performed using the microscopy facility of the Institut Albert Bonniot. This equipment was partly funded by 'Association pour la Recherche sur le Cancer' (Villejuif, France), and the Nanobio program.

\section{References}

1. Alberts, B. et al., Molecular Biology of the Cell, Third edition, Garland, New York (1994).

2. D. Ambrosi, Cellular traction as an inverse problem, SIAM J. Appl. Math, 66:2049-2060 (2006). 
3. Balaban, N.Q., Schwarz, U.S., Riveline, D., Goichberg, P., Tzur, G., Sabanay, I., Mahalu, D., Safran, S., Bershadsky, A., Addadi, L. and Geiger, B., Force and focal adhesion assembly: a close relationship studied using elastic micropatterned substrates, Nat. Cell Biol., 3:466-472 (2001).

4. Boudou, T., Ohayon, J., Picart, C. and Tracqui, P., An extended relationship for the characterization of Youngs modulus and Poissons ratio of tunable polyacrylamide gels, Biorheology, 43:721-728 (2006).

5. Burton, K. and Taylor, D.L., Traction forces of cytokinesis measured with optically modified elastic substrata, Nature, 385:450-454 (1997).

6. Burton, K., Park, J. H. and Taylor, D.L., Keratocytes generate traction forces in two phases, Mol. Biol. Cell, 10:3745-3769 (1999).

7. Butler, J.P., Toli-Nrrelykke, I.M., Fabry, B. and Fredberg, J. J., Traction fields, moments, and strain energy that cells exert on their surroundings, Am. J. Physiol. Cell Physiol., 282, C595-C605 (2002)

8. Choquet, D., Felsenfeld, D.P. and Sheetz, M.P., Extracellular matrix rigidity causes strengthening of integrin-cytoskeleton linkages, Cell, 88:39-48 (1997).

9. Dembo, M. and Wang, Y. L., Stresses at the cell-to-substrate interface during locomotion of fibroblasts, Biophys. J., 76, 2307-2316 (1999).

10. Dembo, M., Oliver, T., Ishihara, A. and Jacobson, K., Imaging the traction stresses exerted by locomoting cells with elastic substratum method, Biophys. J., 70:2008-2022 (1996).

11. Discher, D.E., Janmey, P. and Wang, Y., Tissue cells feel and respond to the stiffness of their substrate, Science, 310:1139-1143 (2005).

12. du Roure, O., Saez, A., Buguin, A., Austin, R.H., Chavrier, P., Silberzan, P. and Ladoux, B., Force mapping in epithelial cell migration, Proc. Natl Acad. Sci. USA, 102:2390-2395 (2005).

13. Engler, A., Bacakova, L., Newman, C., Hategan, A., Griffin, M. and Discher, D., Substrate compliance versus ligand density in cell on gel responses, Biophys. J., 86, 617-628 (2004).

14. Féréol, S., Fodil, R., Labat, B., Galiacy, S., Laurent, V. M., Louis, B., Isabey, D. and Planus, E., Sensitivity of alveolar macrophages to substrate mechanical and adhesive properties, Cell Motil. Cytoskeleton, 63:321-340 (2006).

15. Fichera, G., Existence theorems in elasticity, in Handbuch der Physik, Band VIa/2, edited by C. Truesdell, Springer-Verlag (1972).

16. Galbraith, C. G. and Sheetz, M. P., A micromachined device provides a new bend on fibroblast traction forces, Proc. Natl Acad. Sci. USA, 94:9114-9118 (1997).

17. Harris, A.K., Wild, P. and Stopak, D., Silicone rubber substrata: a new wrinkle in the study of cell locomotion, Science, 208:177-179 (1980).

18. Rasband, W.S., ImageJ, U. S. National Institutes of Health, Bethesda, Maryland, USA, http://rsb.info.nih.gov/ij/ (1997-2007).

19. Landau, L. and Lisfchitz, E., Théorie de l'Élasticité, Editions Mir, Moscou (1967).

20. Lee, J., Leonard, M., Oliver, T., Ishihara, A. and Jacobson, K., Traction Forces Generated by Locomoting Keratocytes, J. Cell Biol., 127:1957-1964 (1994)

21. Lions, J.L., Contrôle optimal de systèmes gouvernés par des équations aux dérivées partielles, Dunod et Gauthier-Villard, Paris (1968).

22. Lo, C.M., Wang, H. B., Dembo, M. and Wang, Y. L., Cell movement is guided by the rigidity of the substrate, Biophys. J., 79:144-152 (2000).

23. Merkel, R., Kirchgessner, N., Cesa, C.M. and Hoffman, B., Cell force microscopy on elastic layers of finite thickness, Biophys. J, 93:3314-3323 (2007).

24. Palecek, S.P., Loftus, J.C., Ginsberg, M.H., Lauffenburger, D.A. and Horwitz, A.F., Integrin-ligand binding properties govern cell migration speed through cell-substratum adhesiveness, Nature, 385:537-540 (1997).

25. Pelham, R.J. and Wang, Y., Cell locomotion and focal adhesions are regulated by substrate flexibility, Proc. Natl Acad. Sci. USA, 94, 13661-13665 (1997).

26. Reinhart-King, C.A., Dembo, M. and Hammer, D.A. The dynamics and mechanics of endothelial cell spreading, Biophys. J., 89:676-689 (2005).

27. Ridley, A.J., Schwartz, M.A., Burridge, K, Firtel, R.A., Ginsberg M.H., Borisy, G., Parsons, J.T., Horwitz, A.R., Cell Migration: Integrating Signals from Front to Back, Science 302:1704-1709 (2003)

28. Rincon, A. and Liu, I.S., On numerical approximation of an optimal control problem in linear elasticity, Divulgaciones Matemáticas, 11:91-107 (2003).

29. Saez, A., Buguin, A., Silberzan, P. and Ladoux, B., Is the Mechanical Activity of Epithelial Cells Controlled by Deformations or Forces? Biophys. J., 89:L52-L54 (2005). 
30. Saez, A., Ghibaudo, M., Buguin, A., Silberzan, P. and Ladoux, B., Rigidity-driven growth and migration of epithelial cells on microstructured anisotropic substrates, Proc. Natl Acad. Sci. USA, 104:8281-8286 (2007).

31. Sabass, B., Gardel, M.L., Waterman, C.M. and Schwarz U.S., High Resolution Traction Force Microscopy Based on Experimental and Computational Advances, Biophys. J. 94:207-220 (2008).

32. Schwarz, U.S., Balaban, N.Q., Riveline, D., Bershadsky, A., Geiger, B. and Safran, S.A., Calculation of forces at focal adhesions from elastic substrate data: the effect of localized force and the need for regularization, Biophys. J., 83:1380-1394 (2002).

33. Tan, J.L., Tien, J., Pirone, D.M., Gray, D.S., Bhadriraju, K. and Chen, C.S., Cells lying on a bed of microneedles: an approach to isolate mechanical force, Proc. Natl Acad. Sci. USA, 100:1484-1489 (2003).

34. Verdier, C., Review. Rheological properties of living materials: From cells to tissues, J. Theor. Medicine, 5, 67-91 (2003).

35. Yeung, T., Georges, P.C., Flanagan, L.A., Marg, B., Ortiz, M., Funaki, M., Zahir, N., Ming, W., Weaver, V. and Janmey, P. A., Effects of substrate stiffness on cell morphology, cytoskeletal structure, and adhesion, Cell Motil Cytoskeleton, 60:24-34 (2005). 